\title{
Asymptotic behavior of Generalized Processor Sharing queues under subexponential assumptions
}

\author{
Marc Lelarge
}

\begin{abstract}
We analyze the behavior of Generalized Processor Sharing (GPS) queues with heavy-tailed service times. We compute the exact tail asymptotics of the stationary workload of an individual class and give new conditions for reduced-load equivalence and induced burstiness to hold. We also show that both phenomena can occur simultaneously. Our proofs rely on the single big event theorem and new fluid limits obtained for the GPS system that can be of interest by themselves.

Keywords: Generalized Processor Sharing; induced burstiness; reduced-load equivalence; subexponential distributions; tail asymptotics; fluid limit.
\end{abstract}

\section{Introduction}

Empirical evidence of the presence of heavy tails in network traffic have stimulated the analysis of queueing systems with subexponential service times. Although the presence of heavy-tailed traffic is widely acknowledged [26], the practical implications for network performance and traffic engineering remain to be fully resolved. The importance of scheduling in the presence of heavy tails was first recognized by Anantharam in [3]. The present paper specifically examines the effectiveness of Generalized Processor Sharing (GPS, precisely described in Section 2.1) with subexponential service times. We compute the exact tail asymptotics of the stationary workload process of each class. Our framework is restricted to instantaneous inputs and extends results of Borst, Boxma and Jelenkovic [11]. Also our proof is new and inspired by the recent work of Baccelli and Foss [6] which has been applied to a variety of networks [8, 12, 7, 20]. All these studies deal with networks which belong to the monotone separable framework. In our GPS system, a single class does not belong to this framework, hence we will need to adapt ideas of [6]. Moreover, we will see that we are able to remove some of the assumptions made in [6] on the service time distribution. To do so, we will need a slight extension of Pakes [23] or Veraverbeke's Theorem [25, 27] which we state as Theorem 3. We will also need to derive the fluid limit of the GPS system in the same way as [17] derived fluid limits for generalized Jackson networks as a first step towards tail asymptotics [7]. Such analysis has been done by Dupuis and Ramanan [13] based on a Skorokhod problem formulation of the GPS model. We choose to give here a probabilistic proof based on a coupling argument. For stationary and ergodic inputs, this proof allows us to construct the stationary workload processes of the stable classes when the system is overloaded, i.e. $\rho>1$. To the best of our knowledge, Theorem 1 is new and extends [13]. Our main result is Theorem 2 which gives the exact tail asymptotics in a unified way: reduced-load equivalence and induced burstiness results of [11] are recovered and extended. Moreover the proof is generic and relies on an extension of the 'single-big-event theorem' well-known for isolated queues $[24,28,6]$. The heurisitic can be stated as follows: a large workload in one queue of the GPS system occurs when one large service time has taken place at one of the queues, while all other service times are close to their means. In the case of subexponential service times, we precisely identify the range of the parameters of the system for which this heuristic can be made rigorous. As observed in [18] (see Section 4.4.4), our results also show that in some cases, this heuristic is not valid and gives only an upper bound on the tail asymptotics. We should also stress that the monotone separable framework has recently been shown to be an efficient tool to derive large deviation results for light-tailed distributions [19,21,22] and we expect that this approach will give new results for GPS system extending [9] to more than 2 classes. 
The paper is structured as follows. In Section 2, we introduce GPS system and construct the stationary GPS system by a coupling argument. We also derive the fluid limits that will be useful for the derivation of the tail asymptotics. In Section 3, we state our main result and compare it with the literature. Its proof is given in Section 4

\section{Notation}

Here and later in the paper, for positive functions $f$ and $g$, the equivalence $f(x) \sim d g(x)$ with $d>0$ means $f(x) / g(x) \rightarrow d$ as $x \rightarrow \infty$. By convention, the equivalence $f(x) \sim d g(x)$ with $d=0$ means $f(x) / g(x) \rightarrow 0$ as $x \rightarrow \infty$, this will be written $f(x)=o(g(x))$.

The tail of the distribution function $F$ is denoted $\bar{F}(x)=1-F(x)$. For a distribution function $F$ on the positive real line with finite first moment $M=\int_{0}^{\infty} \bar{F}(u) d u$, the integrated tail distribution $F^{s}$ of $F$ is defined by

$$
\bar{F}^{s}(x):=1-F^{s}(x)=\min \left\{1, \int_{x}^{\infty} \bar{F}(u) d u\right\} .
$$

We recall here some definitions

Definition 1. A distribution function $F$ on $\mathbb{R}_{+}$is called subexponential if $\overline{F^{* 2}}(x) \sim 2 \bar{F}(x)$.

For basic properties of subexponential distribution see [16, 15, 14].

Definition 2. A positive measurable function $f$ on $[0,+\infty)$ is called regularly varying with index $\alpha \in \mathbb{R}$ $(f \in \mathcal{R}(\alpha))$ if $\lim _{x \rightarrow \infty} \frac{f(t x)}{f(x)}=t^{\alpha}$ for all $t>0$.

Definition 3. A positive measurable function $h$ on $[0,+\infty)$ is called rapidly varying $(h \in \mathcal{R}(-\infty))$ if $\lim _{x \rightarrow \infty} \frac{h(t x)}{h(x)}=0$ for all $t>1$.

For example, Weibull or lognormal random variables have tail distributions that are rapidly varying.

\section{Fluid Limits for GPS Queues}

In this section, we construct the stationary workload at each queue of a GPS system under general stochastic assumptions, namely stationarity and ergodicity. Then we use this result to derive the fluid limits for GPS queues when a big service time occurs.

\subsection{Construction of the Stationary Regime}

Consider the following model of $N$ coupled $G / G / F I F O$ queues. The queues are served in accordance with the Generalized Processor Sharing (GPS) discipline, which operates as follows. Queue $j$ is assigned a weight $\phi^{j}$, with $\sum_{j=1}^{N} \phi^{j}=1$. If all queues are backlogged, then queue $j$ is served at speed $\phi^{j}$. If some of the queues are empty, then the excess capacity is redistributed among the backlogged queues in proportion to their respective weights. All customers within each queue are served in a FIFO order.

More formally we can construct the workload of each queue as follows. We first introduce some notation: customer $n$ arrives in the queue $c_{n}$ at time $T_{n}$ and its service time is $\sigma_{n}$. We will say that this customer is of class $c_{n} \in\{1, \ldots, N\}$ and denote by $\tau_{n}=T_{n+1}-T_{n}>0$ the inter-arrival times. The evolution of the workload processes of each class is given by the following equations:

$$
\begin{aligned}
W^{j}\left(T_{n}\right) & =W^{j}\left(T_{n}-\right)+\sigma_{n} \mathbb{1}_{\left\{c_{n}=j\right\},}, \\
\frac{d W^{j}}{d t}(t) & =-r^{j}(t) \quad \text { for } T_{n} \leq t<T_{n+1}, \\
r^{j}(t) & = \begin{cases}\frac{\phi^{j}}{\sum_{\ell \notin I(t)} \phi^{\ell}} & j \notin I(t), \\
0 & j \in I(t) ;\end{cases} \\
I(t) & =\left\{i, W^{i}(t)=0\right\} .
\end{aligned}
$$


For any initial condition $Y \in \mathbb{R}_{+}^{N}$, we define the workload of each class $W_{Y}^{j}(t)$ for $t \geq 0$ according to Equations $(1,2,3,4)$. We also denote by $W(t)=\sum_{j} W^{j}(t)$ the total workload of the system. Since the GPS discipline is work-conserving, i.e. it serves at the full rate whenever any of the classes is backlogged, the process $W(t)$ is the standard workload process of a single server queue fed by the process $\left\{T_{n}, \sigma_{n}\right\}_{n \in \mathbb{Z}}$.

Assume that the random variables $\left\{\tau_{n}, \sigma_{n}, c_{n}\right\}$ are defined on a common probability space $(\Omega, \mathcal{F}, \mathbb{P}, \theta)$ where $\theta$ is an ergodic, measure-preserving shift transformation, such that $\left(\tau_{n}, \sigma_{n}, c_{n}\right) \circ \theta=\left(\tau_{n+1}, \sigma_{n+1}, c_{n+1}\right)$. Let $\lambda=\mathbb{E}\left[\tau_{0}\right]^{-1}$ be the intensity of arrival process and $\rho=\lambda \mathbb{E}\left[\sigma_{0}\right]$ be the traffic intensity. For example, the process $\left\{T_{n}, \sigma_{n}, c_{n}\right\}$ can be obtained by the superposition of independent renewal point processes of finite intensity (see Section 1.4.2 of [5]) as will be considered in Section 3.

If $\rho<1$, it is easy to construct the stationary workload process of each class. Let $\{W(t)\}, t \in \mathbb{R}$, be the unique stationary workload process of a single server queue with input $\left\{T_{n}, \sigma_{n}\right\}_{n \in \mathbb{Z}}$. The point process $E$ defined by

$$
E(B)=\sum_{n \in \mathbb{Z}} \mathbb{1}_{\left\{T_{n} \in B\right\}} \mathbb{1}_{\left\{W\left(T_{n}-\right)=0\right\}},
$$

counts the points $T_{n}$ at which an arriving customer finds an empty system. Let $\left\{U_{n}\right\}, n \in \mathbb{Z}$, be the sequence of points of $E$, with the usual convention $U_{0} \leq 0<U_{1}$. Then we can construct the unique stationary workload process $\left\{\left(W^{1}(t), \ldots, W^{N}(t)\right)\right\}$ of the GPS queues using Equations $(1,2,3,4)$ on each cycle $\left[U_{n}, U_{n+1}\right)$ with initial condition 0 .

In the case $\rho>1$, it is possible that some classes of the GPS system are still stable. We first give the definition of stability that we consider in this paper.

Definition 4. A stochastic process $X(t)$ is stable if there are an infinite number of negative and positive subscripts $n$ such that $X\left(T_{n}-\right)=0$.

Let $\lambda^{\ell}$ be the intensity of the arrival process $\left\{T_{n}^{\ell}\right\}$ that counts the points of $\left\{T_{n}\right\}$ with mark $c_{n}=\ell$. Let $\rho^{\ell}=\lambda^{\ell} \mathbb{E}\left[\sigma_{0} \mid c_{0}=\ell\right]$ be the traffic intensity of class $\ell$. We have $\rho=\sum_{\ell=1}^{N} \rho^{\ell}$, see Section 1.4.3 of [5]. We assume without loss of generality (w.l.o.g.) that

$$
\frac{\rho^{1}}{\phi^{1}} \leq \cdots \leq \frac{\rho^{N}}{\phi^{N}} .
$$

We define

$$
\begin{gathered}
R_{k}=\frac{1-\sum_{j=1}^{k-1} \rho^{j}}{\sum_{j=k}^{N} \phi^{j}}, \quad K=\max _{k=1, \ldots, N}\left\{k: \frac{\rho^{k}}{\phi^{k}}<R_{k}\right\}, \\
S=\{1, \ldots, K\}, \quad R=\frac{1}{\sum_{j \notin S} \phi^{j}}\left(1-\sum_{j \in S} \rho^{j}\right) .
\end{gathered}
$$

We will show that $S$ is the set of stable queues. This set is empty if and only if $\rho^{1} / \phi^{1} \geq 1$, in this case we take the convention: $K=0$ and $R=1$.

Remark 1. The quantities in Equation (6) were defined in [11]. We will see in Sections 2.2 and 3 that these quantities are crucial for the computation of the tail asymptotics.

For any $k$, we will consider the GPS system indexed by $k$ where classes $i>k$ are always backlogged, i.e. for all $j \leq k$,

$$
\begin{aligned}
\frac{d \bar{W}^{j,[k]}}{d t}(t) & =-r^{j,[k]}(t) \quad \text { for } T_{n} \leq t<T_{n+1} \\
\bar{W}^{j,[k]}\left(T_{n}\right) & =\bar{W}^{j,[k]}\left(T_{n}-\right)+\sigma_{n} \mathbb{1}_{\left\{c_{n}=j\right\}} \\
r^{j,[k]}(t) & =\left\{\begin{array}{cc}
\frac{\phi^{j}}{\sum_{\ell \notin I}{ }^{[k]}(t)} \phi^{\ell} & j \notin I^{[k]}(t), \\
0 & j \in I^{[k]}(t)
\end{array}\right. \\
I^{[k]}(t) & =\left\{i \leq k, \bar{W}^{i,[k]}(t)=0\right\} .
\end{aligned}
$$


Note that for all $\ell>k$, we have $\ell \notin I^{[k]}(t)$ for all $t$, i.e. classes larger than $k+1$ are always backlogged.

Theorem 1. Under previous conditions on the input process $\left\{\tau_{n}, \sigma_{n}, c_{n}\right\}$, we have the following properties:

- there exists a unique stationary stable workload process $\left\{\left(\bar{W}^{1,[K]}(t), \ldots, \bar{W}^{K,[K]}(t)\right)\right\}$ satisfying equations (7-10) for all $t \in \mathbb{R}$ with $k=K$ defined by (6).

- if $K+1 \leq N$, under the additional condition $\frac{\rho^{K+1}}{\phi^{K+1}}>R$, there exists no finite stationary workload process $\left\{W^{i}(t)\right\}, t \in \mathbb{R}$, for any $i \geq K+1$. For any finite initial condition $Y \in \mathbb{R}_{+}^{N}$, we can define the workload of each queue for $t \geq 0$, following equations (1-4), and we have for $i \geq K+1$

$$
W_{Y}^{i}(t) \sim\left(\rho^{i}-\phi^{i} R\right) t \quad \text { as } t \rightarrow \infty .
$$

Remark 2. Note that the condition $K+1 \leq N$ implies $R \leq \frac{\rho^{K+1}}{\phi^{K+1}}$, so that the additional condition in the second bullet item ensures that the queues $i \geq K+1$ are 'strictly' unsatble.

\section{Proof.}

If $\rho<1$, then $K=N$ and the result follows from previous construction on the cycles.

We assume now that $1 \leq K \leq N-1$. The proof will proceed by induction on $k \leq K$ : we show that there exists a unique stationary stable workload process $\left(\bar{W}^{1,[k]}(t), \ldots, \bar{W}^{k,[k]}(t)\right)$ which corresponds to a GPS system where queues $k+1, \ldots, N$ are always backlogged. Moreover $\sum_{i \leq k} \bar{W}^{i,[k]}(t)$ is also a stable process. For $t \geq 0$, we will denote by $\bar{W}_{Y}^{[k]}(t)=\left(\bar{W}_{Y}^{1,[k]}(t), \ldots \bar{W}_{Y}^{k,[k]}(t)\right)$ the process satisfying equations (7), (8), (9) and (10) for $t \geq 0$ and with initial condition $\bar{W}_{Y}^{i,[k]}(0)=Y^{i}$. For any $u \in \mathbb{R}$, we define $\bar{W}_{Y, u}^{[k]}(t)$ similarly but with initial condition $\bar{W}_{Y, u}^{[k]}(u)=Y$ (in particular $\bar{W}_{Y, 0}^{[k]}(t)=\bar{W}_{Y}^{[k]}(t)$ ).

The first step is easy. We have $1 \in S$, hence $\rho^{1}<\phi^{1}$ and $\bar{W}^{1,[1]}(t)$ is simply the workload of a standard $G / G / 1$ queue.

For $k \leq N$, we assume that $\left(\bar{W}^{1,[k-1]}(t), \ldots, \bar{W}^{k-1,[k-1]}(t)\right)$ are given. We consider the random variable

$$
\tilde{r}^{k}(t)=\frac{1}{\sum_{j=1}^{N} \phi^{j} \mathbb{1}_{\left\{j \notin I^{[k-1]}(t)\right\}}} .
$$

We have by construction

$$
\sum_{j=1}^{k-1} \phi^{j} \tilde{r}^{k}(t) \mathbb{1}_{\left\{\bar{W}^{j,[k-1]}(t)>0\right\}}+\tilde{r}^{k}(t) \sum_{j=k}^{N} \phi^{j}=1
$$

and since $\phi^{j} \tilde{r}^{k}(t)$ is exactly the service rate of queue $\bar{W}^{j,[k-1]}$, we have

$$
\mathbb{E}\left[\phi^{j} \tilde{r}^{k}(t) \mathbb{1}_{\left\{\bar{W}^{j,[k-1]}(t)>0\right\}}\right]=\rho^{j} .
$$

In particular, from (11) and (12), we have $\mathbb{E}\left[\tilde{r}^{k}(t)\right]=R_{k}$. For any $Y \geq 0$, we consider the following $G / G / 1$ queue:

$$
\begin{aligned}
& \tilde{W}_{Y}^{k}(t)=Y \\
& \tilde{W}_{Y}^{k}(t)=\left(\tilde{W}_{Y}^{k}\left(T_{n}^{k}-\right)+\sigma_{n}^{k}-\phi^{k} \int_{\left[T_{n}^{k}, t\right)} \tilde{r}^{k}(u) d u\right)^{+}, \quad t \in\left[T_{n}^{k}, T_{n+1}^{k}\right) .
\end{aligned}
$$

By standard Palm calculus (see Section 1.3.1 in [5]), we have

$$
\mathbb{E}\left[\int_{\left[T_{0}^{k}, T_{1}^{k}\right)} \tilde{r}^{k}(u) d u\right]=\frac{R_{k}}{\lambda_{k}}
$$


hence we have $k \in S$ implies that $\tilde{W}^{k}(t)$ is the workload process of a stable $G / G / 1$ queue. Clearly by looking at the service rates, we have that for all $i \leq k-1$,

$$
\begin{aligned}
& \bar{W}_{0,-t}^{i,[k]}(0) \leq \bar{W}_{0,-t}^{i,[k-1]}(0) \stackrel{t \rightarrow \infty}{\longrightarrow} \bar{W}^{i,[k-1]}(0), \\
& \bar{W}_{0,-t}^{k,[k]}(0) \leq \tilde{W}_{0,-t}^{k}(0) \stackrel{t \rightarrow \infty}{\longrightarrow} \tilde{W}^{k}(0) .
\end{aligned}
$$

We are now able to construct a stationary workload process $\bar{W}^{i,[k]}$ of the GPS system where queues $k, \ldots, N$ are always backlogged: note that $\bar{W}_{0,-t}^{i,[k]}(0)$ are increasing in $t$, hence we can take the limit as $t \rightarrow-\infty$ (standard coupling from the past) and the limit is finite thanks to (13) and (14). We now prove uniqueness of this stationary process by a coupling argument. Consider any finite variables $\left(Z_{1}, \ldots, Z_{k}\right)$. Then we have

$$
\sum_{i=1}^{k} \bar{W}_{0}^{i,[k]}(t) \leq \sum_{i=1}^{k} \bar{W}_{Z_{i}}^{i,[k]}(t) \leq \sum_{i=1}^{k-1} \bar{W}_{Z_{i}}^{i,[k-1]}(t)+\tilde{W}_{Z_{k}}^{k}(t)
$$

Let

$$
\nu=\inf \left\{t \geq 0, \sum_{i=1}^{k-1} \bar{W}_{Z_{i}}^{i,[k-1]}(t)+\tilde{W}_{Z_{k}}^{k}(t)=0\right\} .
$$

We clearly have $\mathbb{P}(\nu<\infty)=1$ because

$$
\lim _{t \rightarrow \infty} \frac{1}{t} \int_{0}^{t}\left(\sum_{i=1}^{k-1} r^{i,[k-1]}(u)+\phi^{k} \tilde{r}^{k}(u)\right) d u=\sum_{i=1}^{k-1} \rho^{i}+\phi^{k} R^{k}>\sum_{i=1}^{k} \rho^{i} .
$$

The random time $\nu$ (which depends on the $Z_{i}$ 's) is a coupling time: for all $t \geq \nu$, we have $\bar{W}_{0}^{i,[k]}(t)=$ $\bar{W}_{Z_{i}}^{i,[k]}(t)$. Also by taking $Z_{i}=\bar{W}^{i,[k]}(0)$ constructed above, we see that the processes $\bar{W}_{0}^{i,[k]}$ and $\bar{W}^{i,[k]}$ couple and the uniqueness follows.

It remains to show that queues that are not in $S$ are unstable under the additional condition $\frac{\rho^{K+1}}{\phi^{K+1}}>R$. First assume that $K=N-1$. In this case $\frac{\rho^{N}}{\phi^{N}}>R$ implies that $\rho>1$, since $\rho-1=\rho^{N}-\phi^{N} R$. Now for any finite workload process $\left(\mathcal{W}^{1}(t), \ldots, \mathcal{W}^{N}(t)\right)$ of queues $1, \ldots, N$ for $t \geq 0$, we have for all $i \leq N-1$,

$$
\mathcal{W}^{i}(t) \leq \bar{W}_{\mathcal{W}^{i}(0)}^{i,[N-1]}(t), \quad \forall t \geq 0 .
$$

Moreover standard result for the single server queue gives: $\sum_{i=1}^{N} \mathcal{W}^{i}(t) \sim(\rho-1) t$. This shows that $\mathcal{W}^{N}(t) \rightarrow \infty$ as $t \rightarrow \infty$ and $\mathcal{W}^{N}(t) \sim(\rho-1) t=\left(\rho^{N}-\phi^{N} R\right) t$. In this case, the proposition follows.

We assume now that $\frac{\rho^{K+1}}{\phi^{K+1}}>R$ (with the possible value 0 for $K$, in which case $R=1$ ). This ensures that $\rho>1$. Thanks to the ordering of the subscripts, we have

$$
\frac{\sum_{i=K+1}^{N} \rho^{i}}{\sum_{i=K+1}^{N} \phi^{i}}>R
$$

If we replace the classes $K+1, \ldots N$ by a unique virtual class with weight $\sum_{i=K+1}^{N} \phi^{i}$ and we asume this virtual class is always claiming its full share of the service rate. The service rate received by this virtual class is clearly an upper bound for the sum of the service rates received by the classes $K+1, \ldots N$. Note that the system with this virtual class is the same as the one described above. Hence this virtual class receives mean service rate $\sum_{i=K+1}^{N} \phi^{i} R$ and we have for any finite workload process $\left(\mathcal{W}^{1}(t), \ldots, \mathcal{W}^{N}(t)\right)$ defined on $\mathbb{R}_{+}$(we denote $\mathcal{J}(t)=\left\{i, \mathcal{W}^{i}(t)=0\right\} \subset\{1, \ldots, K\}$ ),

$$
\limsup _{t \rightarrow \infty} \frac{1}{t} \int_{0}^{t} \frac{\phi^{K+1}+\cdots+\phi^{N}}{\sum_{j \in \mathcal{J}(u)} \phi^{j}} d u \leq \sum_{i=K+1}^{N} \phi^{i} R .
$$


In particular, we have

$$
\limsup _{t \rightarrow \infty} \frac{1}{t} \int_{0}^{t} \frac{1}{\sum_{j \in \mathcal{J}(u)} \phi^{j}} d u \leq R
$$

Hence for $i \geq K+1$, we have

$$
\limsup _{t \rightarrow \infty} \frac{1}{t} \int_{0}^{t} \frac{\phi^{i}}{\sum_{j \in \mathcal{J}(u)} \phi^{j}} d u \leq \phi^{i} R<\rho^{i} .
$$

Hence we have $\mathcal{W}^{i}(t) \rightarrow \infty$ as $t \rightarrow \infty$ for $i \geq K+1$ so that we actually have the equality: $\lim _{t \rightarrow \infty} \frac{1}{t} \int_{0}^{t} \frac{\phi^{i}}{\sum_{j \in \mathcal{J}(u)} \phi^{j}} d u=\phi^{i} R$, and then $\mathcal{W}^{i}(t) \sim\left(\rho^{i}-\phi^{i} R\right) t$ as $t \rightarrow \infty$.

Remark 3. 1. We assumed $\frac{\rho^{K+1}}{\phi^{K+1}}>R$ in order to avoid the critical case (corresponding to $\rho=1$ in the single server queue).

2. In the work of Borst, Boxma and Jelenković [11], stability issues are also considered (see their Lemma 4.1). However their notion of stability is weaker than our Definition 4 and it corresponds to the fact that the mean service rate is equal to the rate of the input (see their Remark 4.1). Also, they assume the existence of the mean service rates for each class(see their Appendix A) and then derive the equations they must solve. Our Theorem 1 shows that for stationary ergodic inputs, these mean service rates exist and are equal to $\rho^{i}$ for stable classes (i.e. for $i \in S$ ) and to $\phi^{i} R$ otherwise. This strong result was not needed in [11] for the derivation of the tail asymptotics.

In what follows it will be convenient to consider a GPS system with weights $\phi^{1}, \ldots, \phi^{N}$ (not satisfying Condition (5)) but with greedy classes, i.e. the classes $K+1, \ldots, N$ are continuously claiming their full share of service rate. The other classes behave "normally", i.e. the input processes $\left\{T_{n}^{\ell}, \sigma_{n}\right\}$ satisfy the stationary ergodic conditions. The following result is a direct consequence of Theorem 1.

Proposition 1. For any finite initial conditions $Y \in \mathbb{R}_{+}^{N}$, the process $\sum_{i=1}^{K} W_{Y}^{i}(t)$ is stable if

$$
\max _{j=1}^{K} \frac{\rho^{j}}{\phi^{j}}<\frac{1-\sum_{i=1}^{K} \rho^{i}}{1-\sum_{i=1}^{K} \phi^{i}}:=R .
$$

Moreover there exists a mean service rate for the greedy queues, in the following sense: for any finite initial condition $Y$, let $I_{Y}(t)=\left\{i \leq K, W_{Y}^{i}(t)=0\right\}$, then we have

$$
\lim _{t \rightarrow \infty} \frac{1}{t} \int_{0}^{t} \frac{1}{\sum_{i=1}^{K} \phi^{i} \mathbb{1}_{i \notin I_{Y}(u)}+\sum_{i=K+1}^{N} \phi^{i}}=R .
$$

Hence the mean service rate of greedy queue $j$ is $\phi^{j} R$.

In the case $\max _{j} \frac{\rho^{j}}{\phi^{j}}>R$, at least one of the queues $1, \ldots, K$ is not stable.

\subsection{Rare Events in GPS Queues}

In this section we consider a stable GPS system with $\rho<1$. W.l.o.g we assume that the ordering (5) holds. We are interested in the effect of a very big service time of size $\sigma$ arriving in queue $j$ at time $T_{0}^{j}$. Hence we consider workload process given by equations (1-4) for $t \geq T_{0}^{j}$, with initial condition $\left(W^{1}\left(T_{0}^{j}-\right), \ldots, W^{N}\left(T_{0}^{j}-\right)\right)$, i.e. in the stationary regime but we replace $\sigma_{0}$ by a deterministic value $\sigma$. We assume w.1.o.g that $T_{0}^{j}=0$ and we denote $W^{\{j\}}(\sigma, t)=\left(W^{1,\{j\}}(\sigma, t), \ldots, W^{N,\{j\}}(\sigma, t)\right)$ the corresponding workload process. 
Let $T(\sigma)>0$ be the first time for queue $j$ to empty. On the interval $[0, T(\sigma)]$, the queue $j$ is always backlogged. Hence we are exactly in the situation of Proposition 1 with queue $j$ as greedy queue and if

$$
\max _{i \neq j} \frac{\rho^{i}}{\phi^{i}}>\frac{1-\sum_{i \neq j} \rho^{i}}{1-\sum_{i \neq j} \phi^{i}}
$$

then at least one queue $i \neq j$ begins to grow on this period of time. Hence the situation at time $T(\sigma)$ is that some queues are very big and will remain backlogged for a long period of time. Indeed we are still in the situation of Proposition 1 but this time with a set of greedy queues. We need to introduce some notations in order to describe the situation. Given a set $D=\left\{d_{1}, \ldots, d_{n}\right\} \subset\{1, \ldots, N\}$, with $d_{1} \leq \cdots \leq d_{n}$, consider a GPS system in which queues $\{1, \ldots, N\} \backslash D$ are greedy. We still have $\frac{\rho^{d_{1}}}{\phi^{d_{1}}} \leq \cdots \leq \frac{\rho^{d_{n}}}{\phi^{d_{n}}}$. Hence results of previous section apply ( $\operatorname{set} \rho^{i}=\infty$ for $i \notin D$ ) and we denote

$$
\begin{aligned}
K(D) & =\max _{i=1, \ldots, n}\left\{i: \frac{\rho^{d_{i}}}{\phi^{d_{i}}}<\frac{1-\sum_{\ell=1}^{i-1} \rho^{d_{\ell}}}{\sum_{\ell=i}^{n} \phi^{d_{\ell}}+\sum_{j \notin D} \phi^{j}}\right\} \\
S(D) & =\left\{d_{1}, \ldots, d_{K(D)}\right\}, \\
R(D) & =\frac{1}{\sum_{j \notin S(D)} \phi^{j}}\left(1-\sum_{j \in S(D)} \rho^{j}\right),
\end{aligned}
$$

with the convention $\sum_{-1}^{0}=\sum_{\emptyset}=0$. In words, if queues that are not in $D$ continuously claim their full share of the service rate, then $d_{i} \in S(D)$ implies that class $d_{i}$ is stable and for a class $i \notin D$, it will receive a service rate of $\phi^{i} R(D)$. In the case $D=\{j\}$, we will use the notation $(j)$ instead of $(\{j\})$. It is easy to see that:

$$
\sum_{i=1}^{N} \rho^{i}<1 \Rightarrow R(j) \phi^{j}>\rho^{j} .
$$

Also if

$$
\frac{\rho^{i}}{\phi^{i}}<R(j)
$$

then queue $i$ is insensitive to queue $j$ in the sense that it will remain stable for any value of $\rho^{j}$. Note that it is always the case if $\rho^{i}<\phi^{i}$.

We now analyze the effect of a very big service time in class $j$ when condition (17) is not satisfied. We will attach a superscript ${ }^{\{j\}}$ to the constants that are calculated in this case. We first describe the intuitive picture that will be made rigorous using fluid limits. Because of Equation (16), the output rate of class $j$ is bigger than the input rate. Hence queue $j$ will empty at a time $T(\sigma)$ which is approximately $\frac{\sigma}{\phi^{j} R(j)-\rho^{j}}$. We denote $f_{1}^{\{j\}}=\frac{1}{\phi^{j} R(j)-\rho^{j}}$. Moreover, during this time queues of the classes $k \in\{1, \ldots, N\} \backslash(S(j) \cup\{j\})$ start to build up and reach an approximate level $\sigma z_{1}^{k,\{j\}}$ at time $T(\sigma)$ where we have:

$$
\begin{aligned}
z_{1}^{k,\{j\}} & =\int_{0}^{f_{1}^{\{j\}}}\left(\rho^{k}-\gamma^{k,\{j\}}(u)\right)^{+} d u, \\
\gamma^{k,\{j\}}(t) & =\phi^{k} R(j) \mathbb{1}_{\left\{t \leq f_{1}^{\{j\}}\right\}} .
\end{aligned}
$$

We also denote:

$$
I_{1}=\{1, \ldots, N\} \backslash(S(j) \cup\{j\}), \quad i_{1}^{\{j\}}=j .
$$


At time $T(\sigma)$, queues $k \in I_{1}$ are backlogged and will receive a service rate $\phi^{k} R\left(I_{1}\right)$, whereas other queues including $j$ are stable. Hence for $\ell \geq 1$, we define:

$$
\begin{aligned}
f_{\ell+1}^{\{j\}} & =\min _{i \in I_{\ell}}\left\{\frac{z_{\ell}^{i,\{j\}}}{\phi^{i} R\left(I_{\ell}\right)-\rho^{i}}\right\}+f_{\ell}^{\{j\}}, \\
\left\{i_{\ell+1}^{\{j\}}\right\} & =\arg \min _{i \in I_{\ell}}\left\{\frac{z_{\ell}^{i,\{j\}}}{\phi^{i} R\left(I_{\ell}\right)-\rho^{i}}\right\}, \\
I_{\ell+1} & =I_{\ell} \backslash\left\{i_{\ell}^{\{j\}}\right\}, \\
\gamma^{k,\{j\}}(t) & =\phi^{k} R\left(I_{\ell}\right) \mathbb{1}_{\left\{f_{\ell}^{\{j\}}<t \leq f_{\ell+1}^{\{j\}}\right\}} \\
z_{\ell+1}^{k,\{j\}} & =\int_{0}^{f_{\ell+1}^{\{j\}}}\left(\rho^{k}-\gamma^{k,\{j\}}(u)\right)^{+} d u .
\end{aligned}
$$

The interpretation is the following: at time $f_{\ell+1}^{\{j\}} \sigma$, queues $\left\{i_{\ell+1}^{\{j\}}\right\}$ empty whereas queues in $I_{\ell+1}$ reach levels $z_{\ell+1}^{k,\{j\}} \sigma$. During the time period $\left(f_{\ell}^{\{j\}} \sigma, f_{\ell+1}^{\{j\}} \sigma\right)$, classes in $I_{\ell}$ are continuously backlogged.

For all $k \in\{1, \ldots, N\}$, we defined a function $\gamma^{k,\{j\}}(t)$ for $t \leq \sigma /(1-\rho)$ and we now extend it for values of $t>\sigma /(1-\rho)$ by setting: $\gamma^{k,\{j\}}(t)=\rho^{k}$. We can now define the function

$$
\begin{aligned}
& w^{k,\{j\}}(\sigma, t)=\int_{0}^{t}\left(\rho^{k}-\gamma^{k,\{j\}}(u / \sigma)\right)^{+} d u \quad \forall j \neq k, \\
& w^{j,\{j\}}(\sigma, t)=\left(\sigma+\int_{0}^{t}\left(\rho^{j}-\phi^{j} R(j)\right) d u\right)^{+} .
\end{aligned}
$$

Let $w^{\{j\}}(\sigma, t)=\left(w^{1,\{j\}}(\sigma, t), \ldots, w^{N,\{j\}}(\sigma, t)\right)$ be the multidimensional function. Since the sequence of sets $\left\{I_{\ell}\right\}$ is decreasing, it is easy to see that $R\left(I_{\ell+1}\right)>R\left(I_{\ell}\right)$. Figure 1 illustrates the shape of functions $w^{\{j\}}(\sigma,$.$) for a given \sigma$.

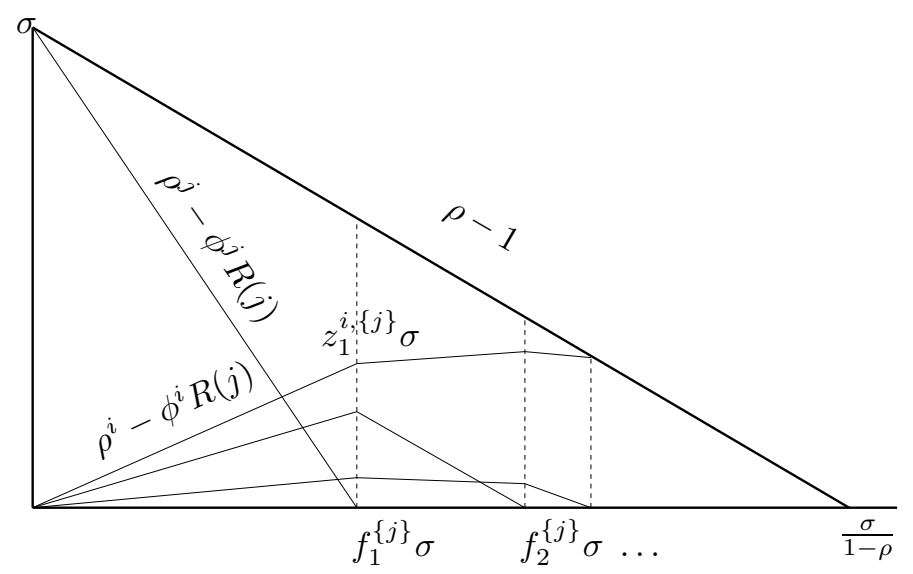

Figure 1: functions $w^{\{j\}}(\sigma,$.$) for fixed \sigma$

Proposition 2. If $\rho<1$, we have for any constant $\alpha, \beta>0$, as $n \rightarrow \infty$,

$$
\sup _{\sigma>n \alpha, t \leq \beta}\left|\frac{W^{\{j\}}(\sigma, n t)-w^{\{j\}}(\sigma, n t)}{n}\right| \rightarrow 0, \quad \text { a.s. }
$$

The proof of this proposition is deferred to Appendix 6. It will be our main tool for the computation of the tail asymptotics presented below. It plays a similar role as Proposition 5.1 in [17] in the study of tail asymptotics for generalized Jackson networks [7]. 


\section{Tails in GPS Queues with Subexponential Service Time Distribu- tions}

\subsection{Stochastic Assumptions and Main Results}

In this section, we restrict the framework of Section 2: we assume that each arrival process $\left\{T_{n}^{j}\right\}_{n \in \mathbb{Z}}$ is a renewal process which is independent of the arrival processes of classes $i \neq j$. We also assume that the sequence of service times of class $j$ denoted by $\sigma_{n}^{j}$ is a sequence of i.i.d. random variables with finite mean. Recall that for each $j \in\{1, \ldots, N\}$, we have $\mathbb{E}\left[T_{1}^{j}-T_{0}^{j}\right]=\frac{1}{\lambda^{j}}<\infty, \rho^{j}=\lambda^{j} \mathbb{E}\left[\sigma_{0}^{j}\right]$ and $\rho=\sum_{j=1}^{N} \rho^{j}$. We assume moreover that for any $i \neq j$, we have $\rho^{i} \neq R(j) \phi^{i}$. Recall that this is always true in the case $i=j$ since $\rho<1$ see Equation (16).

We will consider two cases concerning the distribution of the service times.

Assumption 1. The distribution of the service times of class $i, \mathbb{P}\left(\sigma_{0}^{i}>x\right)=F_{i}(x)$, is such that $F_{i}^{s}$ is subexponential.

The other case considered is the following:

Assumption 2. There exists a distribution function $F$ on $\mathbb{R}_{+}$such that:

1. F has finite first moment $M$.

2. The integrated distribution $F^{s}$ is subexponential.

3. The following equivalence holds when $x$ tends to $\infty$ :

$$
\mathbb{P}\left(\sigma_{0}^{j}>x\right) \sim d^{j} \bar{F}(x),
$$

for all $j=1, \ldots, N$ with $\sum_{j=1}^{N} d^{j}>0$.

Remark 4. Note that in both cases, we did NOT assume that F is subexponential.

We take the notation of Section 2.2 to define the following domains indexed by $i, j \in\{1, \ldots, N\}$ :

$$
\Delta^{i,\{j\}}(x)=\left\{(\sigma, t) \in \mathbb{R}_{+}^{2}, w^{i,\{j\}}(\sigma, t)>x\right\} .
$$

We are now able to state the main result

Theorem 2. Consider a stable GPS system of $N$ queues. We assume that one of the following conditions holds:

- Assumption 1 is satisfied and $\rho^{i}<\phi^{i}$;

- Assumption 2 is satisfied.

Let $W^{i}$ be the stationary workload of queue $i$. When $x \rightarrow \infty$, we have

$$
\mathbb{P}\left(W^{i}>x\right)=\sum_{j=1}^{N} \lambda^{j} \iint_{\left\{(\sigma, t) \in \Delta^{i,\{j\}}(x)\right\}} \mathbb{P}\left(\sigma^{j} \in d \sigma\right) d t+o\left(\bar{F}^{s}(x)\right) .
$$

We will prove Theorem 2 in Section 4. As an intermediate result, we will prove the following theorem which extends Pakes' theorem [23] to a more general setting and might be of interest by itself.

Theorem 3. Let $W$ be the stationary workload of a single server queue fed by the superposition of $N$ independent GI/GI processes with Assumption 2 satisfied. Then we have

$$
\mathbb{P}(W>x) \sim \frac{\lambda}{1-\rho} \mathbb{P}\left(\sigma^{S}>x\right),
$$

where $\lambda$ is the intensity of the arrival process, $\rho$ is the traffic intensity and the distribution of $\sigma^{S}$ is the integrated tail of the (Palm) distribution of the service time.

Remark 5. This result extends Theorem 4.1 of Asmussen, Schmidli and Schmidt [4], in which the arrival process is the superposition of renewal processes but the service times are supposed to be i.i.d. 


\subsection{Reduced-load equivalence and induced burstiness}

We now comment on our main Theorem 2 and show how it extends existing results in the literature. The main results in the literature (see [1] for a survey) reveal a dichotomy in the qualitative behavior, depending on the traffic intensities and the weight values of the various classes:

- reduced-load equivalence occurs when an individual class with subexponential characteristics is served at a constant rate, which is determined by the average rates of the other classes.

- induced burstiness occurs when an individual class is strongly affected by excessive activity of 'heavier'-tailed classes and inherits their traffic characteristics.

The term reduced-load equivalence was first coined in the context of fluid queues with subexponential activity periods [2] and the term induced burstiness first appeared in [10]. We will show that our results allows us to consider mixed cases where both phenomena come into play.

We first give some conditions for reduced-load equivalence to hold.

Proposition 3. We denote by $W^{i, c}$ the stationary workload of a single server queue fed by arrivals of class $i$ with constant service rate $c$. If one of the following conditions holds:

(a) $\rho^{i}<\phi^{i}$ and Assumption 1 holds;

(b) Assumption 2 holds with $d^{j}=0$ for all $j \neq i$;

(c) Assumption 2 holds with $d^{i}>0$ and $\bar{F}^{s} \in \mathcal{R}(-\infty)$;

then the following reduced-load equivalence holds: $\mathbb{P}\left(W^{i}>x\right) \sim \mathbb{P}\left(W^{i, \phi^{i} R(i)}>x\right)$.

This proposition follows easily from Theorem 2 and corresponds (with slightly different conditions) to the reduced-load equivalence proved in [11], Theorems 3.1 and 4.1. Proposition 3 states that the workload of the class $i$ is asymptotically equivalent to that in an isolated system where class $i$ would be served at constant rate $\phi^{i} R(i)$, which is the average rate that class $i$ receives when it continuously claims its full share of the service rate. Asymptotically, the workload of class $i$ is only affected by the traffic characteristics of the other classes through their average rates. In other words, temporary instability caused by other classes (possibly heavier) does not substantially influence the workload of class $i$. Condition $(a)$ ensures stability of class $i$ regardless of the activity of the other classes. Condition $(b)$ ensures that class $i$ is the 'heaviest'tailed class. In the case of Condition $(c)$, the distribution $F^{s}$ is not sufficiently 'heavy' for other classes to impact asymptotically class $i$. This case extends results of [11] and is new.

We now give some cases of induced burstiness.

Proposition 4. If Assumption 2 holds with $d^{i}=0$ and if $\bar{F}^{s} \in \mathcal{R}(-\alpha)$ with $0<\alpha<\infty$, then the following induced burstiness holds: $\mathbb{P}\left(W^{i}>x\right) \sim C^{i} \bar{F}^{s}(x)$, where the constant $C^{i}$ depends only on the parameters $\rho^{j}, \lambda^{j}, d^{j}$, for $j \in\{1, \ldots N\}$ and $\alpha$.

This proposition follows directly from Theorem 2 . We now treat an example in detail.

Example 1. We suppose $N=2$ and Assumption 2 holds with $d^{2}=0, d^{1}=1$ and $\bar{F}^{s} \in \mathcal{R}(-\alpha)$. We also assume that $\frac{\rho^{1}}{\phi^{1}}<1<\frac{\rho^{2}}{\phi^{2}}$. The first inequality is implied by the stability condition $\rho<1$ and we are in the following case: a big service time of class 1 induces an instability of queue 2 . The corresponding fluid limits are depicted in Figure 2.

As stated by Proposition 4, we are in the situation where there is induced burstiness for class 2. The corresponding domain is easy to compute,

$$
\Delta^{2,\{1\}}(x)=\left\{(\sigma, t), t>\frac{x}{\rho^{2}-\phi^{2}}, \sigma>x+(1-\rho) t\right\} .
$$




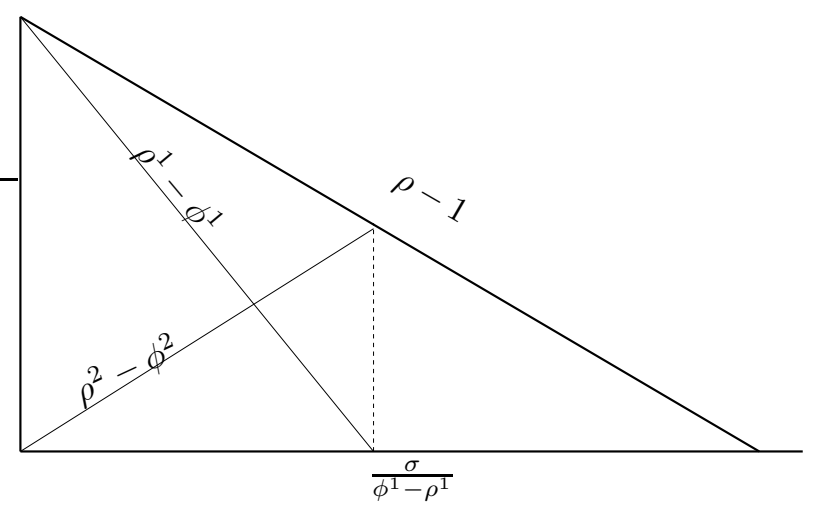

Figure 2: GPS with two classes: big service in class 1

Then we can apply Theorem 2 and we get

$$
\begin{aligned}
\mathbb{P}\left(W^{2}>x\right) & \sim \lambda^{1} \int_{\frac{x}{\rho^{2}-\phi^{2}}}^{\infty} \mathbb{P}\left(\sigma^{1}>x+(1-\rho) t\right) d t \\
& \sim \frac{\lambda^{1}}{1-\rho} \bar{F}^{s}\left(\frac{x\left(1-\rho^{1}\right)}{\rho^{2}-\phi^{2}}\right) \\
& \sim \frac{\lambda^{1}}{1-\rho}\left(\frac{1-\rho^{1}}{\rho^{2}-\phi^{2}}\right)^{-\alpha} \bar{F}^{s}(x) .
\end{aligned}
$$

The computations done in previous example can be made for any value of $N$ and we see that any class that makes the queue $i$ temporary unstable, will contribute to the tail asymptotics of the workload of class $i$ in a non-trivial way given by the shape of the domains. In our framework, Theorem 5.1 of [11] corresponds to cases where only one class can make queue $i$ temporarily unstable and the corresponding domain has a shape similar to the one of $\Delta^{2,\{1\}}(x)$ in previous example. Our Theorem 2 does not require any condition on the shape of the domains and as soon as $N \geq 3$, these domains can have quite intricate shapes (see Lemma 3 in the next section) and more than one class can make class $i$ temporarily unstable.

We now consider a case where service times have similar characteristics in both classes and neither reduced-load equivalence nor induced burstiness happens.

Example 2. Consider Example 1 but with $d^{1}=d^{2}=1$. Then by Theorem 2, we have

$$
\begin{aligned}
\mathbb{P}\left(W^{2}>x\right) & \left.\sim \lambda^{1} \int_{\frac{x}{\rho^{2}-\phi^{2}}}^{\infty} \bar{F}(x+(1-\rho) t) d t+\lambda^{2} \int_{0}^{\infty} \bar{F}(x+(1-\rho) t)\right) d t \\
& \sim\left(\frac{\lambda^{1}}{1-\rho}\left(\frac{1-\rho^{1}}{\rho^{2}-\phi^{2}}\right)^{-\alpha}+\frac{\lambda^{2}}{1-\rho}\right) \bar{F}^{s}(x) .
\end{aligned}
$$

The first part of the right-hand term is exactly the same as in Example 1. The second part of the right-hand term, corresponds to a reduced-load equivalence: class 2 receiving a service rate of $\phi^{2} R(2)=1-\rho^{1}$. We see that in this case both phenomena come into play.

If Assumption 2 holds, we call the set $\left\{i, d_{i}>0\right\}$, the set of dominant classes. In summary, we see that

1. if $\rho^{i}<\phi^{i}$, reduced-load equivalence occurs (provided class $i$ is heavy-tailed);

2. if class $i$ is a dominant class and the corresponding integrated tail is rapidly varying, then reducedload equivalence occurs; 
3. if class $i$ is not a dominant class and the integrated tail of the dominant classes is regularly varying, then induced burstiness occurs;

4. if class $i$ is a dominant class and the integrated tail of the dominant classes is regularly varying, then the tail asymptotics of class $i$ are the sum of two contributions corresponding to both phenomena.

Points 1, 2 and 3 are in accordance with results of [11]. Point 4 is new for a GPS system (similar results were obtained for a queue with unit capacity fed by several On-Off flows [29]).

\section{Proof of the tail asymptotics}

The GPS system does not fit exactly in the framework of [6]. If we consider the global workload (which is a $G / G / 1$ queue), we have a monotone separable network, but the service times are not i.i.d. and it is not possible to directly apply the results of [6]. Hence we need to adapt the argument to our framework in order to derive the single big event theorem for our GPS system. Except in Section 4.4, we assume that Assumption 2 holds.

\subsection{The Single Big Event Theorem}

We first construct an upper bound for $W$. We consider $N$ virtual $G I / G I / 1$ queues with respective input processs $\left\{T_{n}^{j}, \sigma_{n}^{j}\right\}_{n \in \mathbb{Z}}$ and with server capacity $\tilde{r}^{j}=\rho^{j}+\frac{1-\rho}{N}$. We denote by $\tilde{W}^{j}$ the workload at time 0 of these single server queues and $\tilde{W}=\tilde{W}^{1}+\cdots+\tilde{W}^{N}$. More formally, we define

$$
\xi_{n}^{j}=\sigma_{n}^{j}-\tilde{r}^{j} \tau_{n}^{j}, \quad S_{-n}^{j}=\sum_{i=-n}^{0} \xi_{i}^{j}, \quad M^{j}=\sup _{n \geq 0} S_{-n}^{j} .
$$

With these definitions, we have $\tilde{W}^{j}=\left(M^{j}+\tilde{r}^{j} T_{0}^{j}\right)^{+}$, where $T_{0}^{j}<0$ is the last arrival time of class $j$ before time $t=0$.

We have $[23,25]: \mathbb{P}\left(\tilde{W}^{j}>x\right) \sim \frac{N \lambda^{j} d^{j}}{1-\rho} \bar{F}^{s}(x)$. Moreover the random variables $\tilde{W}^{j}$ are independent of each other, hence we have

$$
\mathbb{P}(\tilde{W}>x) \sim \sum_{j=1}^{N} \mathbb{P}\left(\tilde{W}^{j}>x\right) \sim \sum_{j=1}^{N} \frac{N \lambda^{j} d^{j}}{1-\rho} \bar{F}^{s}(x) .
$$

The following corollary follows the line of Corollary 5 of [6].

Corollary 1. For any $x$ and $j=1, \ldots, N$, let $\left\{K_{n, x}^{j}\right\}$ be a sequence of events such that

1. for any $n$, the event $K_{n, x}^{j}$ and the random variables $\left(\sigma_{-n}, c_{-n}\right)$ are independent;

2. $\inf _{n \geq N_{x}} \mathbb{P}\left(K_{n, x}^{j}\right) \rightarrow 1$ as $x \rightarrow \infty$.

For any sequence $\eta_{n} \rightarrow 0$, let

$$
\begin{aligned}
A_{n, x}^{j} & =K_{n, x}^{j} \cap\left\{\sigma_{-n}>x+n\left(\frac{1-\rho}{N \lambda}+\eta_{n}\right), c_{-n}=j\right\} \\
A_{x} & =\bigcup_{j=1}^{N} \bigcup_{n \geq N_{x}} A_{n, x}^{j} .
\end{aligned}
$$

Then as $x \rightarrow \infty$,

$$
\mathbb{P}(\tilde{W}>x) \sim \mathbb{P}\left(\tilde{W}>x, A_{x}\right) \sim \mathbb{P}\left(A_{x}\right) \sim \sum_{j=1}^{N} \sum_{n \geq N_{x}} \mathbb{P}\left(A_{n, x}^{j}\right) .
$$




\section{Proof.}

The proof follows the one of Corollary 5 of [6]. First note that

$$
\begin{aligned}
\sum_{j=1}^{N} \sum_{n \geq N_{x}} \mathbb{P}\left(A_{n, x}^{j}\right) & =\sum_{j=1}^{N} \sum_{n \geq N_{x}} \mathbb{P}\left(K_{n, x}^{j}\right) \mathbb{P}\left(\sigma_{-n}>x+n\left(\frac{1-\rho}{N \lambda}+\eta_{n}\right), c_{-n}=j\right) \\
& \sim \sum_{j=1}^{N} \sum_{n \geq N_{x}} \frac{\lambda^{j}}{\lambda} \mathbb{P}\left(\sigma_{-n}^{j}>x+n\left(\frac{1-\rho}{N \lambda}+\eta_{n}\right)\right) \\
& \sim \sum_{j=1}^{N} \frac{\lambda^{j}}{\lambda} \frac{N \lambda}{1-\rho} d^{j} \bar{F}^{s}(x)=\sum_{j=1}^{N} \frac{N \lambda^{j} d^{j}}{1-\rho} \bar{F}^{s}(x) .
\end{aligned}
$$

Thus, if the sequences $\left\{K_{n, x}\right\}$ and $\left\{\eta_{n}\right\}$ are such that, for all sufficiently large $x$,

1. the events $A_{n, x}^{j}$ are disjoint for all $n \geq N_{x}$;

2. $A_{n, x}^{j} \subset\{\tilde{W}>x\}$ for all $n \geq N_{x}$;

then

$$
\begin{aligned}
\mathbb{P}(\tilde{W}>x) & \geq \mathbb{P}\left(\tilde{W}>x, A_{x}\right)=\mathbb{P}\left(A_{x}\right) \\
& =\sum_{j=1}^{N} \sum_{n \geq N_{x}} \mathbb{P}\left(A_{n, x}^{j}\right) \sim \sum_{j=1}^{N} \frac{N \lambda^{j} d^{j}}{1-\rho} \bar{F}^{s}(x) .
\end{aligned}
$$

Combining with (19), we get the equivalence (20).

We now construct two specific sequences $\left\{K_{n, x}^{j}\right\}$ and $\left\{\eta_{n}\right\}$ satisfying conditions 1 and 2 above and the conditions of the corollary.

We define the following function $C^{j}(n)=\sum_{k=-n}^{0} \mathbb{1}_{\left\{c_{k}=j\right\}}-1$. On the event $\left\{c_{-n}=j\right\}$, we have $T_{-n}=T_{-C^{j}(n)}^{j}, \sigma_{-n}=\sigma_{-C^{j}(n)}^{j}$. We can find a non-increasing sequence $\epsilon_{n} \rightarrow 0$ such that $n \epsilon_{n} \rightarrow \infty$ and such that the probabilities of the following events tend to 1 as $n \rightarrow \infty$,

$$
\begin{aligned}
L_{n, x} & =\left\{\left|\frac{S_{-k}^{j}}{k}-\frac{\rho-1}{N \lambda^{j}}\right| \leq \epsilon_{k}, N_{x} \leq k \leq C^{j}(n-1), 1 \leq j \leq N\right\}, \\
M_{n}^{j} & =\left\{\left|\frac{C^{j}(n-1)}{n}-\frac{\lambda^{j}}{\lambda}\right| \leq \epsilon_{n}\right\}, \\
N_{n}^{j} & =\left\{\left|T_{0}^{j}\right| \leq \frac{n \epsilon_{n}}{\tilde{r}^{j}}\right\} .
\end{aligned}
$$

Hence the event $K_{n, x}^{j}=L_{n, x} \cap M_{n}^{j} \cap N_{n}^{j}$ satisfies the conditions of the corollary. Moreover on the event $\left\{c_{-n}=j\right\}$, we have $C^{j}(n)=C^{j}(n-1)+1$ and, $S_{-C^{j}(n)}^{j}=\sigma_{-n}+S_{-C^{j}(n-1)}^{j}$.

Now if we take $\eta_{n}=\sqrt{\epsilon_{n}}$, we have

$$
\begin{aligned}
\tilde{W} & \geq S_{-C^{j}(n)}^{j}-n \epsilon_{n} \\
& >x+n\left(\frac{1-\rho}{N \lambda}+\eta_{n}\right)+n\left(\frac{\lambda^{j}}{\lambda}-\epsilon_{n}\right)\left(\frac{\rho-1}{N \lambda^{j}}-\epsilon_{\left(\lambda^{j} / \lambda\right) n-1}\right)-n \epsilon_{n},
\end{aligned}
$$

and we see that for sufficiently large $n$, we have $\tilde{W}>x$. The fact that the events $A_{n, x}^{j}$ are disjoint follows from the fact that for sufficiently large $x$, we have $\epsilon_{N_{x}} \leq(1-\rho) /\left(N \lambda^{j}\right)$. Indeed on the event $A_{n, x}^{j}$, we have $S_{-C^{j}(n)}^{j}>x$ and $S_{-C^{j}(n)+1}^{j} \leq\left(C^{j}(n)-1\right)\left((\rho-1) /\left(N \lambda^{j}\right)+\epsilon_{N_{x}}\right) \leq 0$. The events $\left\{S_{n}^{j}>x\right\} \cup\left\{S_{n-1}^{j} \leq 0\right\}$ are clearly disjoint in $n$. It is also easy to see that the events $A_{n, x}^{j}$ are disjoint in $j$. The end of the proof, i.e. showing that the corollary is true for any sequence $K_{n, x}^{j}$ follows exactly the line of the proof of Corollary 5 of [6] and is omitted.

From this corollary we derive the following proposition 
Proposition 5. For any $x$ and $j=1, \ldots, N$, let $\left\{K_{n, x}^{j}\right\}$ be a sequence of events such that

1. for any $n$, the event $K_{n, x}^{j}$ and the random variables $\left(\sigma_{-n}, c_{-n}\right)$ are independent;

2. $\inf _{n \geq N_{x}} \mathbb{P}\left(K_{n, x}^{j}\right) \rightarrow 1$ as $x \rightarrow \infty$.

For any sequence $\eta_{n} \rightarrow 0$, let

$$
\begin{aligned}
A_{n, x}^{j} & =K_{n, x}^{j} \cap\left\{\sigma_{-n}>x+n\left(\frac{1-\rho}{N \lambda}+\eta_{n}\right), c_{-n}=j\right\} \\
A_{x} & =\bigcup_{j=1}^{N} \bigcup_{n \geq N_{x}} A_{n, x}^{j} .
\end{aligned}
$$

Then for any random variable $W \leq \tilde{W}$, we have as $x \rightarrow \infty$,

$$
\begin{aligned}
\mathbb{P}(W>x) & =\mathbb{P}\left(W>x, A_{x}\right)+o\left(\bar{F}^{s}(x)\right) \\
& =\sum_{j=1}^{N} \sum_{n \geq N_{x}} \mathbb{P}\left(W>x, A_{n, x}^{j}\right)+o\left(\bar{F}^{s}(x)\right) .
\end{aligned}
$$

Proof.

We have

$$
\begin{aligned}
\mathbb{P}(W>x) & =\mathbb{P}\left(W>x, A_{x}\right)+\mathbb{P}\left(W>x, A_{x}^{c}\right) \\
& \leq \mathbb{P}\left(W>x, A_{x}\right)+\mathbb{P}\left(\tilde{W}>x, A_{x}^{c}\right),
\end{aligned}
$$

but thanks to previous corollary we have that $\mathbb{P}\left(\tilde{W}>x, A_{x}^{c}\right)=o\left(\bar{F}^{s}(x)\right)$. Hence we have

$$
\mathbb{P}\left(W>x, A_{x}\right) \leq \mathbb{P}(W>x) \leq \mathbb{P}\left(W>x, A_{x}\right)+o\left(\bar{F}^{s}(x)\right),
$$

which gives (21). The end of the proof is the same as the one of last corollary.

\subsection{Proof of Theorem 3.}

First note that $\tilde{W} \geq W$. Hence we can apply previous proposition, with

$$
K_{n, x}^{j}=\left\{\left|\frac{S_{-k}}{k}-\frac{\rho-1}{\lambda}\right| \leq \epsilon_{k}, N_{x} \leq k \leq n-1,\left|T_{0}\right| \leq n \epsilon_{n}\right\},
$$

where $S_{-k}=\sum_{i=-k}^{0} \sigma_{i}-\tau_{i}$. On the event $A_{n, x}^{j}$, we have $W=\sigma_{-n}+S_{-n+1}+T_{0}^{A}$, hence

$$
\begin{aligned}
\sum_{j=1}^{N} \sum_{n \geq N_{x}} \mathbb{P}\left(W>x, A_{n, x}^{j}\right) & \sim \sum_{j=1}^{N} \sum_{n \geq N_{x}} \mathbb{P}\left(\sigma_{-n}>x+n\left(\frac{1-\rho}{\lambda}+2 \epsilon_{n}\right), c_{-n}=j\right) \\
& \sim \frac{\lambda}{1-\rho} \bar{F}^{s}(x) .
\end{aligned}
$$

\subsection{Computation of the Exact Asymptotics when Assumption 2 holds}

We have to find a sequence of events $\left\{K_{n, x}^{j}\right\}$ in order to compute the following sum

$$
S^{i,\{j\}}=\sum_{n \geq N_{x}} \mathbb{P}\left(W^{i}>x, K_{n, x}^{j}, \sigma_{-n}>x+n\left(\frac{1-\rho}{N \lambda}+\eta_{n}\right), c_{-n}=j\right)
$$

A first case is easy: when queue $i$ remains stable even if queue $j$ is continuously backlogged. 
Lemma 1. Assume that

$$
\frac{\rho^{i}}{\phi^{i}}<R(j)
$$

Then we have $S^{i,\{j\}}=o\left(\bar{F}^{s}(x)\right)$.

\section{Proof.}

Under condition (23), we know thanks to Proposition 1, that the stationary workload of queue $i$ exists when queue $j$ is continuously backlogged. We denote this workload $W^{i}(j)$. We have $W^{i} \leq W^{i}(j)<\infty$, and $W^{i}(j)$ is clearly independent of $\left(T_{n}^{j}, \sigma_{n}^{j}\right)$. Hence we have

$$
\begin{aligned}
S^{i,\{j\}} & =\sum_{n \geq N_{x}} \mathbb{P}\left(W^{i}>x, K_{n, x}^{j}, \sigma_{-n}>x+n\left(\frac{1-\rho}{N \lambda}+\eta_{n}\right), c_{-n}=j\right) \\
& \leq \mathbb{P}\left(W^{i}(j)>x\right) \sum_{n \geq N_{x}} \mathbb{P}\left(\sigma_{-n}>x+n\left(\frac{1-\rho}{N \lambda}+\eta_{n}\right), c_{-n}=j\right) \\
& =o\left(\bar{F}^{s}(x)\right) .
\end{aligned}
$$

We consider now the case $\frac{\rho^{i}}{\phi^{i}}>R(j)$. In this case when queue $j$ experiences a long backlog (due to a very big service time), queue $i$ is no longer stable and the fluid limit corresponding to this queue is no longer 0 . The remaining steps of the proof of Theorem 2 are similar to those of Section 3 in [7].

Let $\epsilon_{n}$ be some sequence of positive real numbers, we define

$$
K_{n}^{j}=\left\{\sup _{\substack{\sigma>n \frac{1-\rho}{N \lambda} \\ t \leq 2 a}}\left|\frac{W^{\{j\}}\left(\sigma, T_{-n}+n t\right)-w^{\{j\}}(\sigma, n t)}{n}\right| \leq \epsilon_{n},\left|\frac{T_{-n}}{n}+a\right| \leq \epsilon_{n}\right\} .
$$

Thanks to the results of Section 2.2, we have the following lemma

Lemma 2. Let $\left\{K_{n}^{j}\right\}$ be the sequence of events defined above. $K_{n}^{j}$ and the random variables $\sigma_{-n}$ and $c_{-n}$ are independent. There exists a sequence $\epsilon_{n} \rightarrow 0$ such that we have $\mathbb{P}\left(K_{n}^{j}\right) \rightarrow 1$ as $n \rightarrow \infty$.

On the event $K_{n}^{j} \cap\left\{\sigma_{-n}>x+n\left(\frac{1-\rho}{N \lambda}\right), c_{-n}=j\right\}$, we have (thanks to the continuity of $w^{i,\{j\}}$ ),

$$
W^{i}=w^{i,\{j\}}\left(\sigma_{-n}, n a\right)+n \eta_{n}, \quad \text { with } \eta_{n} \text { a r.v. such that }\left|\eta_{n}\right| \leq \epsilon_{n} .
$$

We will need the following lemma on the shape of the domain $\Delta^{i,\{j\}}(x)$.

Lemma 3. There exist constants $\left\{\alpha_{k}^{i,\{j\}}, \beta_{k}^{i,\{j\}}, \gamma_{k}^{i,\{j\}}\right\}_{0 \leq k \leq \ell}$ with $\alpha_{0}^{i,\{j\}}<\alpha_{1}^{i,\{j\}}<\cdots<\alpha_{\ell}^{i,\{j\}}$, $\beta_{k}^{i,\{j\}} \leq 1$, such that

$$
\Delta^{i,\{j\}}(x)=\bigcup_{k=0}^{\ell}\left\{\alpha_{k}^{i,\{j\}} x \leq t<\alpha_{k+1}^{i,\{j\}}, \sigma>\frac{x}{\beta_{k}^{i,\{j\}}}+t \gamma_{k}^{i,\{j\}}\right\}
$$

with $\alpha_{\ell+1}^{i,\{j\}}=+\infty$. Moreover, we have

$$
\Delta^{i,\{i\}}(x)=\left\{\sigma>x+\left(\phi^{i} R(i)-\rho^{i}\right) \lambda t\right\}
$$

This lemma follows directly from the definition of the function $w^{i,\{j\}}$. 
Following exactly the steps of the proof of Theorem 2.1 in [7], we can show that

$$
\begin{aligned}
S^{i,\{j\}}(x) & \sim \sum_{k=0}^{\ell} \sum_{\alpha_{k}^{i,\{j\}}} \mathbb{P}\left(\sigma_{-n}>\frac{x}{\beta_{k}^{i\{j\}}}+n a \gamma_{k}^{i,\{j\}}, c_{-n},\{,\{j\}\right. \\
& =\frac{\lambda^{j}}{\lambda a} \sum_{k=0}^{\ell} \sum_{\alpha_{k}^{i,\{j\}}} \mathbb{P}\left(\sigma^{j}>\frac{x}{\beta_{k}^{i\{j\}}}+n \gamma_{k}^{i,\{j\}}\right) \\
& =\lambda^{j} \sum_{k=0}^{\ell} \sum_{\alpha_{k}^{i,\{j\}}} \sum_{x \leq n<\alpha_{k+1}^{i,\{j\}}} \mathbb{P}\left(\sigma^{j}>\frac{x}{\beta_{k}^{i,\{j\}}}+n \gamma_{k}^{i,\{j\}}\right) .
\end{aligned}
$$

This term is of order $d^{j} \bar{F}^{s}\left(x / \beta_{0}^{i,\{j\}}\right)$ and hence $o\left(\bar{F}^{s}(x)\right)$ as soon as $F^{s}$ is rapidly varying. Summing over $j$, we obtain the equality (18) of the Theorem, which concludes the proof.

\subsection{Tail asymptotics when Assumption 1 holds}

The stationary workload of the $G I / G I / 1$ queue with input process $\left\{T_{n}^{i}, \sigma_{n}^{i}\right\}_{n \in \mathbb{Z}}$ and service rate $\phi^{i}$ is clearly a stable upper-bound for $W^{i}$. The proof then follows from the same arguments as above.

\section{Acknowledgements}

I am thankful to the referee for useful suggestions and to François Baccelli and Serguei Foss for initiating me to this topic.

\section{References}

[1] S. Aalto, U. Ayesta, S. Borst, V. Misra, and R. Núnez-Queija. Beyond processor sharing. SIGMETRICS Perform. Eval. Rev., 34(4):36-43, 2007.

[2] R. Agrawal, A. M. Makowski, and P. Nain. On a reduced load equivalence for fluid queues under subexponentiality. Queueing Systems Theory Appl., 33(1-3):5-41, 1999. Queues with heavy-tailed distributions.

[3] V. Anantharam. Scheduling strategies and long-range dependence. Queueing Systems Theory Appl., 33(1-3):73-89, 1999. Queues with heavy-tailed distributions.

[4] S. Asmussen, H. Schmidli, and V. Schmidt. Tail probabilities for non-standard risk and queueing processes with subexponential jumps. Adv. in Appl. Probab., 31(2):422-447, 1999.

[5] F. Baccelli and P. Brémaud. Elements of queueing theory, volume 26 of Applications of Mathematics (New York). Springer-Verlag, Berlin, second edition, 2003. Palm martingale calculus and stochastic recurrences, Stochastic Modelling and Applied Probability.

[6] F. Baccelli and S. Foss. Moments and tails in monotone-separable stochastic networks. Ann. Appl. Probab., 14(2):612-650, 2004.

[7] F. Baccelli, S. Foss, and M. Lelarge. Tails in generalized Jackson networks with subexponential service-time distributions. J. Appl. Probab., 42(2):513-530, 2005.

[8] F. Baccelli, M. Lelarge, and S. Foss. Asymptotics of subexponential max plus networks: the stochastic event graph case. Queueing Syst., 46(1-2):75-96, 2004. 
[9] D. Bertsimas, I. C. Paschalidis, and J. N. Tsitsiklis. Large deviations analysis of the generalized processor sharing policy. Queueing Systems Theory Appl., 32(4):319-349, 1999.

[10] S. Borst, O. Boxma, and P. Jelenković. Induced burstiness in generalized processor sharing queues with long-tailed traffic flows. Proc. of the 37th Annual Allerton Conf. on Communication, Control and Computing, pages 316-325, 1999.

[11] S. Borst, O. Boxma, and P. Jelenković. Reduced-load equivalence and induced burstiness in GPS queues with long-tailed traffic flows. Queueing Syst., 43(4):273-306, 2003.

[12] A. B. Dieker and M. Lelarge. Tails for (max, plus) recursions under subexponentiality. Queueing Syst., 53(4):213-230, 2006.

[13] P. Dupuis and K. Ramanan. A Skorokhod problem formulation and large deviation analysis of a processor sharing model. Queueing Systems Theory Appl., 28:109-124, 1998.

[14] P. Embrechts, C. Klüppelberg, and T. Mikosch. Modelling Extremal Events for Insurance and Finance. Springer Verlag, 2003.

[15] C. M. Goldie and C. Klüppelberg. Subexponential distributions. In A practical guide to heavy tails (Santa Barbara, CA, 1995), pages 435-459. Birkhäuser Boston, Boston, MA, 1998.

[16] C. Klüppelberg. Subexponential distributions and integrated tails. J. Appl. Probab., 25(1):132-141, 1988.

[17] M. Lelarge. Fluid limit of generalized Jackson queueing networks with stationary and ergodic arrivals and service times. J. Appl. Probab., 42(2):491-512, 2005.

[18] M. Lelarge. Rare events in networks. PhD thesis, École polytechnique, 2005.

[19] M. Lelarge. Tail asymptotics for monotone-separable networks. J. Appl. Probab., 44(2):306-320, 2007.

[20] M. Lelarge. Packet reordering in networks with heavy-tailed delays. Math. Methods Oper. Res., 67(2):341-371, 2008.

[21] M. Lelarge. Tail asymptotics for discrete event systems. Discrete Event Dyn. Syst., 18(4):563-584, 2008.

[22] M. Lelarge, Z. Liu, and C. H. Xia. Asymptotic tail distribution of end-to-end delay in networks of queues with self-similar cross traffic. In INFOCOM, 2004.

[23] A. G. Pakes. On the tails of waiting-time distributions. J. Appl. Probability, 12(3):555-564, 1975.

[24] S. Resnick and G. Samorodnitsky. Activity periods of an infinite server queue and performance of certain heavy tailed fluid queues. Queueing Systems Theory Appl., 33(1-3):43-71, 1999. Queues with heavy-tailed distributions.

[25] N. Veraverbeke. Asymptotic behaviour of Wiener-Hopf factors of a random walk. Stochastic Processes Appl., 5(1):27-37, 1977.

[26] W. Willinger, V. Paxson, R. H. Riedi, and M. S. Taqqu. Long-range dependence and data network traffic. In Theory and applications of long-range dependence, pages 373-407. Birkhäuser Boston, Boston, MA, 2003.

[27] S. Zachary. A note on Veraverbeke's theorem. Queueing Syst., 46(1-2):9-14, 2004.

[28] A. P. Zwart. Queueing Systems with Heavy Tails. PhD thesis, Eindhoven University of Technology, 2001.

[29] B. Zwart, S. Borst, and M. Mandjes. Exact asymptotics for fluid queues fed by multiple heavy-tailed on-off flows. Ann. Appl. Probab., 14(2):903-957, 2004. 


\section{Appendix: proof of Proposition 2}

We consider a sequence $\sigma^{n}$ such that $\sigma^{n}>n \alpha$. We suppose that $\frac{\sigma^{n}}{n} \rightarrow \sigma \leq+\infty$. We will show that

$$
\sup _{0 \leq t \leq \beta}\left|\frac{W^{\{j\}}\left(\sigma^{n}, n t\right)-w^{\{j\}}\left(\sigma^{n}, n t\right)}{n}\right| \rightarrow 0,
$$

which is sufficient to prove the proposition. For simplicity, we denote $W_{n}^{\{j\}}(t)=W^{\{j\}}\left(\sigma^{n}, t\right)$ and $w_{n}^{\{j\}}(t)=w^{\{j\}}\left(\sigma^{n}, t\right)$.

We first assume that $\sigma<\infty$. Let $T_{1}^{n}$ be the first positive time at which queue $j$ becomes empty, i.e. queue $j$ is backlogged on $\left[0, T_{1}^{n}\right]$. Hence we have thanks to the result on the mean service rate of Proposition 1 ,

$$
\lim _{n \rightarrow \infty} \frac{W_{n}^{j,\{j\}}\left(T_{1}^{n}\right)}{n}=\sigma+\left(\rho^{j}-\phi^{j} R(j)\right)\left(\lim _{n \rightarrow \infty} \frac{T_{1}^{n}}{n}\right)=0
$$

from which we derive $\lim _{n \rightarrow \infty} \frac{T_{1}^{n}}{n}=\sigma /\left(\phi^{j} R(j)-\rho^{j}\right)$. Now for $0 \leq t \leq T_{1}^{n} / n$, we can apply Proposition 1 and we have

$$
\begin{aligned}
& \frac{W_{n}^{\ell,\{j\}}(n t)}{n} \rightarrow\left(\rho^{\ell}-\phi^{\ell} R(j)\right)^{+} t, \quad \forall \ell \neq j, \\
& \frac{W_{n}^{j,\{j\}}(n t)}{n} \rightarrow \sigma+\left(\rho^{j}-\phi^{j} R(j)\right) t .
\end{aligned}
$$

We have shown in the case $\sigma<\infty$ that for all $j$,

$$
\sup _{0 \leq t \leq f_{1}^{\{j\}} \sigma}\left|\frac{W_{n}^{\{j\}}(n t)-w_{n}^{\{j\}}(n t)}{n}\right| \rightarrow 0 .
$$

Moreover, we see that at time $T_{1}^{n}$, the queues $k \in I_{1}$ are backlogged. Define $T_{n}^{2}$ as the first time at which one of these queues becomes empty. Using Proposition 1 in the same manner, we obtain that $T_{2}^{n} / n \rightarrow \sigma f_{2}^{\{j\}}$ and that,

$$
\sup _{0 \leq t \leq f_{2}^{\{j\}} \sigma}\left|\frac{W_{n}^{\{j\}}(t)-w_{n}^{\{j\}}(n t)}{n}\right| \rightarrow 0 .
$$

Hence in the case $\sigma<\infty$, the proposition follows by iterating the same kind of arguments.

In the case $\sigma=+\infty$, since $T_{1}^{n} \geq \sigma^{n}$, we have for sufficiently large $n$, we have $T_{1}^{n} \geq n \beta$. Hence for all $k \neq j$, we have with the same argument as above that

$$
\sup _{0 \leq t \leq \beta}\left|\frac{W_{n}^{k,\{j\}}(n t)-w_{n}^{k,\{j\}}(n t)}{n}\right| \rightarrow 0,
$$

and for $k=j$, we have for all $t \leq \beta$,

$$
\frac{W_{n}^{j,\{j\}}(n t)-\sigma^{n}}{n} \rightarrow\left(\rho^{j}-\phi^{j} R(j)\right) t .
$$

This concludes the proof. 